\title{
Accuracy Tests Of American Put Valuation Models For Pharmaceutical Equity Options
}

Yong H. Kim, (Email: yhk@hansei.ac.kr), Hansei University, South Korea Sangwoo Heo, (Email: heo@usi.edu), University of Southern Indiana

Peter Cashel-Cordo, (Email: cashel@usi.edu), University of Southern Indiana

Yong S. Jang, (Email: ysjang@mk.co.kr), Maeil Business Newspaper, South Korea

\begin{abstract}
This study compares the performance of the Macmillan (1986), Barone-Adesi and Whaley (1987) MBAW model, Ju and Zhong (1999) MQuad model, Black-Scholes model and Put-Call Parity in pricing American put options of pharmaceutical companies. These are evaluated using actual option prices for three companies over 2000 to 2005, as opposed to the previous use of generated binomial option pricing data. We compare the forecasting accuracy by maturity, moneyness, and variance estimate. Contrary to Ju and Zhong (1999), we find that the MBAW outperforms the other models for at-the-money, and out-of-the-money options. The MQuad model performs best for in-the-money options. However, in this case both the MBAW and MQuad models estimates are very similar. Our results are consistent irrespective of option maturities and volatility estimates. These findings raise questions regarding the practice of using actual prices as the true value, compared to the previous results that use simulated prices.
\end{abstract}

\section{INTRODUCTION}

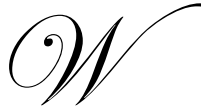

ith the advent of Black and Scholes (BS) (1973) seminal work, a significant portion of finance literature has examined the forecasting accuracy of option pricing models. With the exception of Zhu (2006), American option pricing models have lacked mathematically closed formula, Zhu closed-form exact solution is written in the form of a Taylor's series expansion containing infinitely many terms. Consequently, for ease of implementation and effective computation researchers still must rely on approximation methods. One of well-known analytical approximation has been developed by MacMillan (1986) and Barone-Adesi and Whaley (1987). However, because their model is a quadratic approximation, there can be serious mispricing. More recently, Ju and Zhong (1999) ${ }^{1}$ modified the MBAW method. They report that their method performs better than MBAW for options with very short and very long maturities reducing the pricing error for intermediatematurity options. There are, however, very few empirical studies with actual American put option data using the MQuad method.

In this study, we conduct an accuracy test of the three models, BS, MBAW, and MQuad. These models are tested by variance estimate (implied volatility and historical volatility), by moneyness, and by option maturity (less than one year and greater than or equal to one year) in pricing American put options. We also include American put prices that are obtained from put-call parity by assuming that European put-call parity holds and that investors are rational. For accuracy measures, the mean absolute percent error (MAPE) and the root mean squared error (RMSE) are used. Contrary to the Ju and Zhong, we use actual daily put-call options data. It is for three pharmaceutical companies, Merck (MRK), Pfizer (PFE), and Schering-Plough (SGP) traded on the Chicago Board of Trade Option Exchange (CBOE), from November 3, 2000 to December 6, 2005.

The balance of the paper is organized as follows. In section II, we review MBAW and MQuad method in detail and the related literature and develop our testable issues. Section III describes the data and methodology. Empirical results are reported in section IV. The final section concludes the paper and presents some suggestions for future research.

\footnotetext{
${ }^{1}$ Hereafter, we refer to the pricing model developed by MacMillan (1986) and Barone-Adesi and Whaley (1987) as MBAW and pricing model developed by Ju and Zhong (1999) as MQuad.
} 


\section{MODEL REVIEW}

Over the past few decades, pricing American options has been a topic of interest. The literature on option pricing valuation models is well documented in Broadie and Detemple's (2004) survey paper. Unfortunately, they do not include analytic approximation methods in American options. In this section, we present the detail of MBAW model and MQuad model that we implement in this study and discuss the related literature.

\section{II.A. MBAW Method}

Barone-Adesi and Whaley (1987) provide an accurate and relatively simple method for valuing American options by adopting a quadratic approximation approach originally developed by MacMillan (1986).

Let

$S$ : Current Stock Price,

$X$ : Exercise (Strike) Price,

$r$ : Current risk-free interest rate,

$\sigma:$ Security price volatility,

$T$ : Time to expiration,

$\delta$ : Current dividend yield (for dividend paying stocks).

Assuming American option put price $P=P_{E}($ European Put Price $)+\rho($ Premium Variable $)$, the premium variable $\rho$ satisfies the Black-Sholes differential equation

$$
\frac{1}{2} S^{2} \frac{\partial^{2} \rho}{\partial S^{2}} \sigma^{2}-r \rho+(r-\delta) S \frac{\partial \rho}{\partial S}+\frac{\partial \rho}{\partial t}=0
$$

Let $\rho=K(T) \Psi(S, K)$. After necessary changes,

$$
K=1-e^{-r T}, M_{1}=2 r / \sigma^{2}, \text { and } M_{2}=2(r-\delta) / \sigma^{2},
$$

$\Psi(S, K)$ satisfies

$$
S^{2} \frac{\partial^{2} \Psi}{\partial S^{2}}+M_{2} S \frac{\partial \Psi}{\partial S}-\frac{M_{1} \Psi}{K}-(1-K) M_{1} \frac{\partial \Psi}{\partial K}=0 .
$$

Assuming the last term in (2) to be zero, then the equation (2) becomes

$$
S^{2} \frac{\partial^{2} \Psi}{\partial S^{2}}+M_{2} S \frac{\partial \Psi}{\partial S}-M_{1} \frac{\Psi}{K}=0 \text {. }
$$

Because equation (3) is a second-order ordinary differential equation, the solutions can be found in terms of a power of $S$. The solution for an American put price is given as follows.

$$
P(S, T)=P_{E}(S, T)+A\left(S / S^{*}\right)^{\lambda} \text {, if } S>S^{*} \text { or } P(S, T)=X-S \text {, if } S \leq S^{*} \text {, }
$$

where

$$
A=-\left(S^{*} / \lambda\right)\left\{1-e^{(-\delta T)} N\left[-d_{1}\left(S^{*}\right)\right]\right\}, \text { and } \lambda=\frac{1}{2}\left\{1-M_{2}-\sqrt{\left(1-M_{2}\right)^{2}+\frac{4 M_{1}}{K}}\right\} \text {. }
$$

The critical stock price $S^{*}$ is obtained by solving the equation

$$
X-S^{*}=P_{E}\left(S^{*}\right)-\left\{1-e^{-\delta T} N\left[-d_{1}\left(S^{*}\right)\right]\right\} S^{*} / \lambda .
$$


The MBAW method loses accuracy due to the assumptions that $\rho=K(T) \Psi(S, K)$ and $(1-K) M_{1} \frac{\partial \Psi}{\partial K}=0$. The second assumption makes sense because $(1-K) M_{1} \frac{\partial \Psi}{\partial K} \rightarrow 0$ as $T \rightarrow 0$ or $T \rightarrow \infty$. That is, MBAW approximation is relatively accurate for very short or very long maturities but there is possibility of serious mispricing for the intermediate cases.

They conduct a simulation study comparing their model's performance to other numerical approaches. Simulation results show that the values generated by the quadratic approximation method are virtually identical to those values predicted by other more sophisticated numerical approaches. Hence, their quadratic approximation method is considerably more computationally efficient than other numerical methods. Furthermore, their analytic approximation valuing American options can be easily applied to value other derivative securities.

Overdahl (1988) uses actual market options exercise data of Treasury bond futures contracts to test a model developed by Barone-Adesi and Whaley (1987). They found significant deviations between the model's predictions and the data and concluded that the Barone-Adesi and Whaley's analytic approximation formula yields biased estimates at which an American option should be exercised for a critical stock price.

Loudon (1990) performed an empirical test of the pricing of put options traded on the Australian Options Market. He compared the predictive ability of the American put valuation formulas using MBAW and Johnson (1983) and Blomeyer (1986) models to the BS European model. He confirms that there exist significant and systematic pricing errors in those American put option pricing models, although the American model prices, particularly MBAW estimation, are significantly closer to market prices than are the BS European model prices.

\section{II.B. MQuad Method}

Ju and Zhong (1999) modified the assumptions of MBAW by including a correction term for the early exercise premium. If the early exercise premium of MBAW is denoted by $K(T) \Psi_{1}(S, K)$, then the correction term is added by $K(T) \Psi_{2}(S, K)$. Let $\Psi=\Psi_{1}+\Psi_{2}$. Equation (2) then becomes

$$
S^{2} \frac{\partial^{2} \Psi_{1}}{\partial S^{2}}+M_{2} S \frac{\partial \Psi_{1}}{\partial S}-\frac{M_{1} \Psi_{1}}{K}+S^{2} \frac{\partial^{2} \Psi_{2}}{\partial S^{2}}+M_{2} S \frac{\partial \Psi_{2}}{\partial S}-\frac{M_{1} \Psi_{2}}{K}-(1-K) M_{1}\left(\frac{\partial \Psi_{1}}{\partial K}+\frac{\partial \Psi_{2}}{\partial K}\right)=0
$$

Equation (5) is then divided into two equations,

$$
S^{2} \frac{\partial^{2} \Psi_{1}}{\partial S^{2}}+M_{2} S \frac{\partial \Psi_{1}}{\partial S}-\frac{M_{1} \Psi_{1}}{K}=0
$$

and

$$
S^{2} \frac{\partial^{2} \Psi_{2}}{\partial S^{2}}+M_{2} S \frac{\partial \Psi_{2}}{\partial S}-\frac{M_{1} \Psi_{2}}{K}-(1-K) M_{1}\left(\frac{\partial \Psi_{1}}{\partial K}+\frac{\partial \Psi_{2}}{\partial K}\right)=0
$$

The solution to the equation (6) is $\Psi_{1}=A\left(S / S^{*}\right)^{\lambda}$, consistent with the MBAW solution in equation (4). The complete solution to the equation (5) depends on the solution of the remaining equation (7).

The MBAW method captures most of the early exercise premium by assuming that $\Psi_{2}$ is relatively small compared to $\Psi_{1}$. Letting $\Psi_{2}=\varepsilon \Psi_{1}$, where $\varepsilon$ is small, then $\varepsilon$ satisfies the PDE

$$
S^{2} \frac{\partial^{2} \varepsilon}{\partial S^{2}}+\left(2 S^{2} \frac{1}{\Psi_{1}} \frac{\partial \Psi_{1}}{\partial S}+M_{2} S\right) \frac{\partial \varepsilon}{\partial S}-(1-K) M_{1}\left((1+\varepsilon) \frac{1}{\Psi_{1}} \frac{\partial \Psi_{1}}{\partial K}+\frac{\partial \varepsilon}{\partial K}\right)=0
$$


To solve (8), Ju and Zhong assume that $\frac{\partial \varepsilon}{\partial K}=0$ and $(1+\varepsilon)$ is a constant. Because $\Psi_{1}=A\left(S / S^{*}\right)^{\lambda}$, equation (8) can be written as

$S^{2} \frac{\partial^{2} \varepsilon}{\partial S^{2}}+(2 \lambda+\beta) S \frac{\partial \varepsilon}{\partial S}-(1-K) M_{1}(1+\varepsilon)\left[\frac{A^{\prime}(K)}{A(K)}+\lambda^{\prime}(K) \log \left(S / S^{*}\right)-\lambda(K) \frac{1}{S^{*}} \frac{\partial S^{*}}{\partial K}\right]=0$.

Letting $Y=\log \left(S / S^{*}\right), \quad \varepsilon=B(K) Y^{2}+C(K) Y$, and by matching the coefficients in (9), the price of an American option is approximated by

$P(S, T)=P_{E}(S, T)+\frac{K(T) A(K)\left(S / S^{*}\right)^{\lambda(K)}}{1-\chi}$ if $S>S^{*}$ or $P(S, T)=X-S$ if $S<S^{*}$,

where $K(T) A(K)=X-S^{*}-P_{E}(S, T)$ and $S^{*}$ solves the equation

$X-S^{*}=P_{E}\left(S^{*}, T\right)-\left\{1-e^{-\delta T} N\left[-d_{1}\left(S^{*}\right)\right]\right\} S^{*} / \lambda$, and $\chi=b\left[\log \left(S / S^{*}\right)\right]^{2}+c \log \left(S / S^{*}\right)$

where

$b=\frac{(1-K) M_{1} \lambda^{\prime}(K)}{2\left(2 \lambda+M_{2}-1\right)}, \quad c=-\frac{(1-K) M_{1}}{2 \lambda+M_{2}-1}\left[\frac{1}{K A(K)} \frac{\partial P_{E}\left(S^{*}, T\right)}{\partial K}+\frac{1}{K}+\frac{\lambda^{\prime}(K)}{2 \lambda+M_{2}-1}\right]$,

$\lambda^{\prime}(K)=\frac{M_{1}}{K^{2} \sqrt{\left(1-M_{2}\right)^{2}+\frac{4 M_{1}}{K}}}$, and

$\frac{\partial P_{E}\left(S^{*}, T\right)}{\partial K}=\frac{S^{*} e^{-d_{1}\left(S^{*}\right) / 2} \sigma e^{(r-\delta) T}}{2 r \sqrt{2 \pi T}}+\frac{\delta S^{*} N\left[-d_{1}\left(S^{*}\right)\right] e^{(r-\delta) T}}{r}-X N\left[-d_{2}\left(S^{*}\right)\right]$.

They demonstrate in the simulation that the MQuad method is computationally as efficient as MBAW and more efficient than other numerical approaches. Furthermore, it is more accurate than the equally efficient MBAW model. They claim that their approximation gives better results "for very short- and very long-maturity options, and substantially reduces the pricing errors for intermediate-maturity options". These results however were obtained from their use of generated prices as the true option value from a binomial tree model with 10,000 time steps and pre-assigned parameter values.

There are very few empirical studies with actual American put option data using the MQuad method. In our study, we employed Ju and Zhong's model but we used actual daily data for pharmaceutical equity options and compared them to the model's generated prices. Since pricing ability crucially depends on unobserved volatility $\sigma$, it is important to use as good an estimator of volatility as possible. Therefore, we use two different volatility measures; implied volatility and historical volatility. 


\section{DATA AND METHODOLOGY}

This study uses daily data of three pharmaceutical companies; Merck (MRK), Pfizer (PFE), and ScheringPlough (SGP) put-call options traded on the Chicago Board of Trade Option Exchange (CBOE) from November 3 of 2000 to December 6 of 2005 . $^{2}$ The data is filtered using the following criteria. To make one-day implied volatility available the first entry of each new put option is deleted. We also deleted observations if the put option prices and call options prices are less than $\$ 0.50$ to offset transaction costs. Finally, the American put prices had to satisfy the following no arbitrage boundary condition:

$$
C_{\text {actual }}-S+X e^{-r T} \leq P_{\text {model }} \leq C_{\text {actual }}-S+X
$$

After filtering we were left with 73,054 usable put-call pairs (26,693 observations for Merck, 28,478 observations for Pfizer, and 19,883 for Schering-Plough). Table 1 summarizes how the number of observations evolves during the screening process.

We then calculated the pricing errors of the MBAW, MQuad, BS models, and Put-call parity. We calculated pricing errors using two estimates for volatility. Implied volatility over the past one day was recovered from the BS model and historical volatility was obtained with daily index returns for the previous 180 trading days. In-the-money, at-the-money, and out-of-the-money options are defined as options with the moneyness value (S/X) of less than 0.98 , between 0.98 and 1.02 , and greater than 1.02 , respectively. The price of options used in this study is the average between bid and ask quotes.

To evaluate the accuracy of each model, we employ two measures: mean absolute percentage error with respect to option price (MAPE), and root mean squared error (RMSE), given by the following:

$$
M A P E=\frac{1}{N} \sum \frac{\left|P_{\text {actual }}-P_{\text {model }}\right|}{P_{\text {actual }}} \times 100(\%), \quad R M S E=\sqrt{\frac{1}{N} \sum\left(P_{\text {actual }}-P_{\text {model }}\right)^{2}},
$$

where $P_{\text {actual }}$ is the actual option price, $P_{\text {model }}$ is the model-generate price and $\mathrm{N}$ is the number of observations. ${ }^{3}$

\section{EMPIRICAL RESULTS}

The results of the accuracy tests for BS, MBAW, MQuad, and Put-Call Parity of all usable option data for the three pharmaceutical companies combined are reported in Table 2 across two dimensions, moneyness and maturity. The results are obtained by comparing model predicted values to observed market values using mean absolute percentage error (MAPE) and root mean squared error (RSME). The boldfaced numbers in the table show the most accurate model for each category of implied volatility (IV) and historical volatility (HV). These results are included in Table 2.

\footnotetext{
2 All data are purchased from www.ivolatility.com.

3 These computations are performed on Linux with a Xeon processor using Mathematica.
} 
Table 1

\begin{tabular}{|l|c|c|c|c|}
\hline & Total & MRK & PFE & SGP \\
\hline Retrieved observations & 245,227 & 85,071 & 92,805 & 67,351 \\
\hline After deleting lag 1 & 242,893 & 84,229 & 91,940 & 66,724 \\
\hline After deleting put option price <\$0.50 & 182,706 & 66,041 & 66,167 & 50,498 \\
\hline After deleting call option price <\$0.50 & 97,401 & 36,869 & 34,457 & 26,075 \\
\hline After screening arbitrage condition: & & & & \\
Final sample & 73,054 & 24,693 & 28,478 & 19,883 \\
maturity < 1 year & 39,670 & 12,103 & 15,979 & 11,588 \\
maturity < 1 year & 33,384 & 12,590 & 12,499 & 8,295 \\
\hline In-the-money & 40,650 & 15,291 & 14,292 & 11,067 \\
maturity < 1 year & 20,075 & 6,775 & 7,282 & 6,01 \\
maturity $\geq 1$ year & 20,575 & 8,516 & 7,010 & 5,049 \\
\hline At-the-money & 6,013 & 2,094 & 2,642 & 1,277 \\
maturity < 1 year & 4,312 & 1,377 & 1,923 & 1,012 \\
maturity $\geq 1$ year & 1,701 & 717 & 719 & 265 \\
\hline Out-of-the-money & 26,391 & 7,308 & 11,544 & 7,539 \\
maturity < 1 year & 15,283 & 3,951 & 6,774 & 4,558 \\
maturity $\geq 1$ year & 11,108 & 3,357 & 4,770 & 2,981 \\
\hline
\end{tabular}

First note that across the board for all four models, using implied volatility produced better accuracy than using historical volatility which is consistent with previous studies. Consequently, we will focus our discussion to the results using implied volatility.

Secondly, in all cases both the MBAW and MQuad models outperform the BS and Put-Call Parity models. This unsurprising result is due to the latter two models were developed for pricing European options whereas the MBAW and MQuad models are more flexible and are designed for pricing the early exercise allowed in American options. With these results in mind we further limit our discussion to comparing the MBAW and MQuad pricing performance.

Referring to the full sample results in Table 2, we compare pricing errors in the context of maturity and moneyness. Ju and Zhong (1999) show in their simulations that the MQuad model substantially outperforms the MBAW model for short- and long- maturity options. To test this, the full sample is separated into two categories; one with option maturity less than one year $(T<1)$ and the other with option maturity greater than or equal to one year $(T \geq 1){ }^{4}$

Contrary to Ju and Zhong's simulation results we find that our empirical results suggest that option maturity may have an effect on the relative performances between the MBAW and MQuad models. For all maturities, either $T<1, T \geq 1$ or combined (All Maturity), the MBAW model generally outperforms the MQuad model. For All Maturity and $T \geq 1$ the pricing errors between these two models are visibly different with the MBAW model having lower pricing errors in three out of four cases depending on moneyness. In the former case for All Maturity regardless of moneyness (ALL) MBAW pricing error using MAPE is $6.3736 \%$ compared to MQuad's $6.5543 \%$, a difference of $0.18 \%$. In the latter case $T \geq 1$ the pricing errors are $7.8228 \%$ versus $8.2149 \%$ (in MAPE), a $0.39 \%$ difference. The only case in which the MQuad model outperforms the MBAW model is for the inthe-money option case across all maturity categories. The only maturity category where the MBAW model does not clearly dominate the MQuad model is for $T<1$. In this case MBAW dominates again in three of the four cases regarding moneyness but the differences in pricing errors in two cases are very small and in the other two cases the relative performance of the two models is approximately the same. We notice that the gap in pricing errors between MBAW and MQuad becomes larger from short- to long-maturity with the differences in MAPE between the two being $-0.0028 \%$ for $T<1,-0.3921 \%$ for $T \geq 1$. According to Ju and Zhong, the improvement of MQuad over MBAW is enormous for longer maturities. Their forecasting errors were more than five times lower than those

\footnotetext{
${ }^{4}$ A one year demarcation is purely arbitrary as noted in our conclusions.
} 
of MBAW. Our results for these three pharmaceutical companies contradict their findings.

The discrepancy between their results and our result may be due to the fact that Ju and Zhong use only twenty seven options and benchmarks the simulated prices from binomial tree model with 10,000 time steps as the true value, whereas, we use an actual pharmaceutical company option data which consists of 73,054 options. In addition to the choice of data set, Ju and Zhong use pre-assigned volatility $\sigma=0.2,0.3$, and 0.4 whereas we use the implied volatility (and historical volatility with the same results) retrieved from actual market data. In order to solve the differential equation (8), Ju and Zhong assume that $\frac{\partial \varepsilon}{\partial K}=0$ and $(1+\varepsilon)$ is a constant. Unlike the assumption $(1-K) M_{1} \frac{\partial \Psi}{\partial K}=0$ in equation (2), Ju and Zhong's assumption is not justified in term of option forecasting, which could contribute bigger pricing errors.

Furthermore, regardless of maturity we find that the MBAW model yields less pricing error than the MQuad model for at-the-money and out-of-money options, while the MQuad model marginally outperforms the MBAW model for in-the-money options. The differences in pricing errors tend to be larger under the circumstance where the MBAW model dominates. That is to say that for at and out-of-money cases the difference in performance tends to be larger than when the MQuad model dominates for the in-the-money case. The only exception as previously noted is the ambiguous case for maturities less than one year, $T<1$.

Unsurprisingly, both the MBAW and MQuad models perform the best for in-the-money options and the worst for out-of-the-money options. This is due to market values for American put options reflect a time value when the option is out-of-the-money, but market values for in-the-money put options consists of an intrinsic value. Hence out-of-the-money options are subject to more pricing errors than in-the-money options. This result is consistent with Blomeyer and Johnson (1988).

The pricing errors for each company are reported in Tables 3, 4 and 5. The results are very similar to those in Table 2. Table 3 provides pricing errors for All Maturities, Table 4 for maturities less than one year and Table 5 for maturities equal to or greater than a year. With regards to model performance using the two volatility measures the results parallel Table 2 with a couple of minor exceptions. In Table 4 there are two cases in which MQuad performs better for individual firms where MBAW had performed better for the three firms combined. However, the differences or the gap in pricing errors are essentially miniscule. In Table 5 we see the one case in which MBAW rivals MQuad for an in-the-money case, but again the differences between the two is so small as to be meaningless.

Based upon its closed mathematical solution, MQuad may be a more elegant alternative to the BlackScholes partial differential equation than MBAW, however, because the both MBAW and MQuad models implicitly assume that the Black-Scholes model correctly prices European values of American put options, pricing biases reported for both models inherently contain pricing errors resulting from the Black-Scholes model's misspecification of stock price dynamics. The results from our empirical study suggested that both MBAW and MQuad American put valuation models do not fully generate the actual option price and do not overcome the difficulty that lies under Black-Scholes partial differential equation. However, our results suggest that the MBAW model is relatively more efficient in most cases than the MQuad model, and both of these models perform better than the BS, and Put-Call Parity models for American put options for three major pharmaceutical companies.

\section{CONCLUSION AND FUTURE WORK}

This paper evaluates the forecasting accuracy of the MBAW, MQuad, BS, and Put-Call Parity in pricing American put options using three pharmaceutical companies from November 3, 2000 to December 6, 2006. Unlike previous studies (Macmillan, 1986; Barone-Adesi and Whaley, 1987; Ju and Zhong, 1999), we use the actual option data instead of binomial trees simulated data to evaluate and compare performances by option maturities (short-and long-term), moneyness (in-the-money, at-the-money, and out-of-the-money), and volatility estimates (implied volatility and historical volatility). MBAW and MQuad predict put option prices more accurately than BS and PutCall Parity. 
The MBAW model outperforms for all options combined, for at-the-money options, and for out-of-themoney options. The MQuad model marginally performs best for in-the-money options, in particular for maturities of less than one year. These results are consistent irrespective of option maturities, volatility estimates and when disaggregated by firm. These findings refute Ju and Zhong's (1999) results at least for the pharmaceutical industry over this time period. The use of actual option prices over model-generated prices as the true value is evident and beneficial in measuring forecasting errors. Based on the evidence presented in this paper, we believe that the use of actual data as the true option value is a significant improvement over past studies, and can lead to very different results.

Admittedly our results are narrow in scope examining only one industry leading to several questions for future research. These include both the scope of the analysis, i.e., whether the relative performance of different options models holds for a broader range of industries, and methodological issues. With the latter, several questions need to be addressed. The possible sensitivity of the relative performance between the MBAW and MQuad models regarding option maturity sets the stage for further analysis. In essence, how does changing the timeframe affect results? Secondly, the relative performance across moneyness requires further examination. What are the factors that lead to more accurate results for the MQuad model for in-the-money options? Lastly, it may be useful to compare our results to Zhu's (2006) Taylor series expansion model in determining forecasting accuracy of American put option prices. 
Table 2

MAPE and RMSE of American Put Option Models on All Three Companies

\begin{tabular}{|c|c|c|c|c|c|c|c|c|c|c|c|c|c|}
\hline & & \multicolumn{4}{|c|}{ All Maturity } & \multicolumn{4}{|c|}{ Maturity < 1 year } & \multicolumn{4}{|c|}{ Maturity $\geq 1$ year } \\
\hline & & ALL & ITM & ATM & OTM & ALL & ITM & ATM & OTM & ALL & ITM & ATM & OTM \\
\hline \# Observations & & 73,054 & 40,650 & 6,013 & 26,391 & 39,670 & 20,075 & 4,312 & 15,283 & 33,384 & 20,575 & 1,701 & 11,108 \\
\hline \multirow{2}{*}{ Put Call Parity } & MAPE & 23.504 & 13.11 & 17.776 & 40.82 & 15.37 & 8.4486 & 12.306 & 25.326 & 33.171 & 17.659 & 31.643 & 62.137 \\
\hline & RMSE & 0.3863 & 0.1566 & 0.2248 & 0.6 .31 & 0.2214 & 0.0976 & 0.1475 & 0.3295 & 0.518 & 0.1979 & 0.3514 & 0.8455 \\
\hline \multirow{2}{*}{ BS, IV } & MAPE & 10.033 & 9.6635 & 9.1087 & 10.813 & 6.5672 & 5.9253 & 6.4775 & 7.4358 & 14.152 & 13.311 & 15.779 & 15.46 \\
\hline & RMSE & 0.1195 & 0.1131 & 0.1131 & 0.1301 & 0.0793 & 0.0685 & 0.0792 & 0.0916 & 0.1543 & 0.1439 & 0.1711 & 0.1694 \\
\hline \multirow[t]{2}{*}{ MBAW, IV } & MAPE & 6.3736 & 5.1009 & 7.0164 & 8.1875 & 5.154 & 4.1406 & 5.4907 & 6.3902 & 7.8228 & 6.0378 & 10.884 & 10.66 \\
\hline & RMSE & 0.0825 & 0.0651 & 0.0884 & 0.1025 & 0.0669 & 0.052 & 0.0691 & 0.0819 & 0.0978 & 0.0758 & 0.1245 & 0.1255 \\
\hline \multirow[t]{2}{*}{ MQuad, IV } & MAPE & 6.5543 & 4.9856 & 7.1449 & 8.8359 & 5.1568 & 3.9704 & 5.4949 & 6.6197 & 8.2149 & 5.9761 & 11.328 & 11.885 \\
\hline & RMSE & 0.0853 & 0.0644 & 0.09 & 0.1091 & 0.0674 & 0.0506 & 0.0692 & 0.0841 & 0.1026 & 0.0755 & 0.1283 & 0.1362 \\
\hline \multirow[t]{2}{*}{ BS, HV } & MAPE & 16.87 & 9.3278 & 16.81 & 28.5 & 15.761 & 7.5384 & 15.598 & 26.608 & 18.187 & 11.074 & 19.882 & 31.104 \\
\hline & RMSE & 0.254 & 0.1264 & 0.2348 & 0.376 & 0.2557 & 0.1132 & 0.2288 & 0.3717 & 0.2519 & 0.1381 & 0.2494 & 0.3819 \\
\hline \multirow[t]{2}{*}{ MBAW, HV } & MAPE & 14.873 & 6.8239 & 16.014 & 27.01 & 15.283 & 7.0179 & 15.491 & 26.08 & 14.386 & 6.6345 & 17.339 & 28.29 \\
\hline & RMSE & 0.243 & 0.1075 & 0.2288 & 0.3657 & 0.2541 & 0.1115 & 0.2291 & 0.3694 & 0.2291 & 0.1034 & 0.2282 & 0.3605 \\
\hline \multirow[t]{2}{*}{ MQuad, HV } & MAPE & 15 & 6.7852 & 16.037 & 27.418 & 15.341 & 7.0032 & 15.488 & 26.252 & 14.595 & 6.5725 & 17.429 & 29.021 \\
\hline & RMSE & 0.2448 & 0.107 & 0.2289 & 0.3691 & 0.2547 & 0.1114 & 0.229 & 0.3705 & 0.2324 & 0.1026 & 0.2286 & 0.3671 \\
\hline
\end{tabular}


Table 3

MAPE and RMSE of American Put Option Models on each company

\begin{tabular}{|c|c|c|c|c|c|c|c|c|c|c|c|c|c|}
\hline & & \multicolumn{4}{|c|}{ MRK } & \multicolumn{4}{|c|}{ PFE } & \multicolumn{4}{|c|}{ SGP } \\
\hline & & ALL & ITM & ATM & OTM & ALL & ITM & ATM & OTM & ALL & ITM & ATM & OTM \\
\hline \# Observations & & 24,693 & 15,291 & 2,094 & 7,308 & 28,478 & 14,292 & 2,642 & 11,544 & 19,883 & 11,067 & 1,277 & 7,539 \\
\hline \multirow[t]{2}{*}{ Put Call Parity } & MAPE & 30.912 & 17.009 & 25.163 & 61.651 & 22.685 & 11.676 & 15.443 & 37.973 & 15.478 & 9.5771 & 10.492 & 24.986 \\
\hline & RMSE & 0.5145 & 0.1982 & 0.3046 & 0.8863 & 0.3437 & 0.136 & 0.1854 & 0.5104 & 0.2246 & 0.1091 & 0.1211 & 0.3362 \\
\hline \multirow[t]{2}{*}{ BS, IV } & MAPE & 12.947 & 12.304 & 12.447 & 14.429 & 9.2007 & 8.6542 & 8.0598 & 10.138 & 7.6069 & 7.3145 & 5.8038 & 8.3415 \\
\hline & RMSE & 0.1491 & 0.1395 & 0.1484 & 0.1675 & 0.1087 & 0.1003 & 0.965 & 0.1206 & 0.0894 & 0.0844 & 0.0694 & 0.0991 \\
\hline \multirow[t]{2}{*}{ MBAW, IV } & MAPE & 8.3587 & 6.8473 & 9.9457 & 11.067 & 5.9476 & 4.3721 & 6.1271 & 7.857 & 4.5184 & 3.6291 & 4.0531 & 5.9027 \\
\hline & RMSE & 0.10479 & 0.0847 & 0.1195 & 0.1342 & 0.0744 & 0.0532 & 0.0727 & 0.0947 & 0.0584 & 0.0449 & 0.0492 & 0.0750 \\
\hline \multirow[t]{2}{*}{ MQuad, IV } & MAPE & 8.549 & 6.733 & 10.109 & 11.902 & 6.1209 & 4.2413 & 6.2365 & 8.4214 & 4.6976 & 3.5324 & 4.164 & 6.4985 \\
\hline & RMSE & 0.1074 & 0.0839 & 0.1215 & 0.1415 & 0.0777 & 0.0523 & 0.0741 & 0.1012 & 0.0611 & 0.0444 & 0.0504 & 0.0808 \\
\hline \multirow[t]{2}{*}{ BS, HV } & MAPE & 19.123 & 11.078 & 21.877 & 35.165 & 17.048 & 8.9829 & 14.714 & 27.566 & 13.817 & 7.3546 & 12.836 & 23.47 \\
\hline & RMSE & 0.3006 & 0.1511 & 0.3132 & 0.4789 & 0.2448 & 0.1185 & 0.1868 & 0.3499 & 0.1974 & 0.095 & 0.163 & 0.2916 \\
\hline \multirow[t]{2}{*}{ MBAW, HV } & MAPE & 16.524 & 7.9096 & 20.893 & 33.297 & 15.505 & 7.0794 & 14.172 & 26.242 & 11.916 & 4.9938 & 11.823 & 22.093 \\
\hline & RMSE & 0.2936 & 0.1352 & 0.3116 & 0.4745 & 0.2316 & 0.0976 & 0.1772 & 0.3367 & 0.182 & 0.0701 & 0.1495 & 0.2763 \\
\hline \multirow[t]{2}{*}{ MQuad, HV } & MAPE & 16.647 & 7.8534 & 20.933 & 33.817 & 15.653 & 7.0735 & 14.178 & 26.613 & 12.02 & 4.9372 & 11.855 & 22.445 \\
\hline & RMSE & 0.2944 & 0.1345 & 0.3114 & 0.4766 & 0.234 & 0.0974 & 0.1775 & 0.3408 & 0.1845 & 0.0697 & 0.1501 & 0.2807 \\
\hline
\end{tabular}

Table 4

MAPE and RMSE of American Put Option Models on each company (Maturity < 1 year)

\begin{tabular}{|c|c|c|c|c|c|c|c|c|c|c|c|c|c|}
\hline & & \multicolumn{4}{|c|}{ MRK } & \multicolumn{4}{|c|}{ PFE } & \multicolumn{4}{|c|}{ SGP } \\
\hline & & ALL & ITM & ATM & OTM & ALL & ITM & ATM & OTM & ALL & ITM & ATM & OTM \\
\hline \# Observations & & 12,013 & 6,775 & 1,377 & 3,951 & 15,979 & 7,282 & 1,923 & 6,774 & 11,588 & 6,018 & 1,012 & 4,558 \\
\hline \multirow[t]{2}{*}{ Put Call Parity } & MAPE & 20.553 & 10.882 & 16.5 & 38.549 & 14.793 & 7.7773 & 11.271 & 23.334 & 10.752 & 6.5215 & 8.5662 & 16.824 \\
\hline & RMSE & 0.2997 & 0.1244 & 0.195 & 0.4852 & 0.1996 & 0.0878 & 0.1295 & 0.2844 & 0.1381 & 0.071 & 0.0954 & 0.1995 \\
\hline \multirow[t]{2}{*}{ BS, IV } & MAPE & 8.5257 & 7.6608 & 8.61 & 9.9794 & 6.1106 & 5.4298 & 5.9511 & 6.8877 & 5.1514 & 4.5711 & 4.5763 & 6.0453 \\
\hline & RMSE & 0.1002 & 0.0865 & 0.1035 & 0.1191 & 0.073 & 0.0619 & 0.0701 & 0.084 & 0.0608 & 0.0508 & 0.053 & 0.0733 \\
\hline \multirow[t]{2}{*}{ MBAW, IV } & MAPE & 6.777 & 5.5365 & 7.4659 & 8.6641 & 4.7997 & 3.6943 & 5.0034 & 5.9301 & 3.9475 & 3.1093 & 3.7291 & 5.1028 \\
\hline & RMSE & 0.0852 & 0.0677 & 0.0917 & 0.107 & 0.061 & 0.0452 & 0.0603 & 0.0746 & 0.0509 & 0.0373 & 0.0449 & 0.0655 \\
\hline \multirow[t]{2}{*}{ MQuad, IV } & MAPE & 6.7785 & 5.3504 & 7.4776 & 8.9837 & 4.8061 & 3.5206 & 5.0002 & 6.1329 & 3.9464 & 2.9609 & 3.7374 & 5.2941 \\
\hline & RMSE & 0.0857 & 0.0662 & 0.0918 & 0.1098 & 0.0616 & 0.0437 & 0.0602 & 0.0767 & 0.0512 & 0.0361 & 0.045 & 0.0671 \\
\hline \multirow[t]{2}{*}{ BS, HV } & MAPE & 19.662 & 9.12 & 21.01 & 37.362 & 15.401 & 7.4417 & 13.661 & 24.451 & 12.151 & 5.875 & 11.914 & 20.491 \\
\hline & RMSE & 0.3447 & 0.1474 & 0.3235 & 0.5387 & 0.2243 & 0.102 & 0.1744 & 0.3144 & 0.1743 & 0.0754 & 0.1511 & 0.2544 \\
\hline \multirow[t]{2}{*}{ MBAW, HV } & MAPE & 18.941 & 8.1644 & 20.815 & 36.768 & 15.261 & 7.5116 & 13.782 & 24.011 & 11.491 & 5.1298 & 11.496 & 19.89 \\
\hline & RMSE & 0.3459 & 0.1471 & 0.3263 & 0.5406 & 0.2213 & 0.101 & 0.1736 & 0.3098 & 0.1692 & 0.069 & 0.1465 & 0.2484 \\
\hline \multirow[t]{2}{*}{ MQuad, HV } & MAPE & 18.992 & 8.1273 & 20.828 & 36.983 & 15.341 & 7.5444 & 13.765 & 24.169 & 11.528 & 5.0829 & 11.497 & 20.045 \\
\hline & RMSE & 0.346 & 0.147 & 0.3262 & 0.5409 & 0.2223 & 0.1011 & 0.1735 & 0.3115 & 0.17 & 0.0686 & 0.1465 & 0.2501 \\
\hline
\end{tabular}


Table 5

MAPE and RMSE of American Put Option Models on each company (Maturity $\geq 1$ year)

\begin{tabular}{|c|c|c|c|c|c|c|c|c|c|c|c|c|c|}
\hline & & \multicolumn{4}{|c|}{ MRK } & \multicolumn{4}{|c|}{ PFE } & \multicolumn{4}{|c|}{ SGP } \\
\hline & & ALL & ITM & ATM & OTM & ALL & ITM & ATM & OTM & ALL & ITM & ATM & OTM \\
\hline \# Observations & & 12,590 & 8,516 & 717 & 3,357 & 12,499 & 7,010 & 719 & 4,770 & 8,295 & 5,094 & 265 & 2,981 \\
\hline \multirow[t]{2}{*}{ Put Call Parity } & MAPE & 40.871 & 21.883 & 41.8 & 88.841 & 32.775 & 15.725 & 26.599 & 58.762 & 22.08 & 13.219 & 17.844 & 37.466 \\
\hline & RMSE & 0.6578 & 0.2412 & 0.445 & 1.197 & 0.4671 & 0.1724 & 0.2855 & 0.7181 & 0.307 & 0.1417 & 0.1895 & 0.4744 \\
\hline \multirow[t]{2}{*}{ BS, IV } & MAPE & 17.197 & 16.003 & 19.817 & 19.667 & 13.151 & 12.004 & 13.7 & 14.755 & 11.037 & 10.584 & 10.491 & 11.852 \\
\hline & RMSE & 0.1842 & 0.1702 & 0.2091 & 0.2107 & 0.1417 & 0.1286 & 0.1451 & 0.1587 & 0.1183 & 0.112 & 0.1118 & 0.1289 \\
\hline \multirow[t]{2}{*}{ MBAW, IV } & MAPE & 9.8798 & 7.8901 & 14.708 & 13.894 & 7.4151 & 5.0762 & 9.1325 & 10.594 & 5.3159 & 4.2487 & 5.2906 & 7.1258 \\
\hline & RMSE & 0.1207 & 0.0961 & 0.16 & 0.1604 & 0.0887 & 0.0604 & 0.0984 & 0.1174 & 0.0676 & 0.0525 & 0.0631 & 0.0876 \\
\hline \multirow[t]{2}{*}{ MQuad, IV } & MAPE & 10.251 & 7.8328 & 15.162 & 15.337 & 7.8017 & 4.99 & 9.5432 & 11.671 & 5.747 & 4.2136 & 5.7933 & 8.3402 \\
\hline & RMSE & 0.1248 & 0.0958 & 0.1641 & 0.1715 & 0.0943 & 0.0599 & 0.1023 & 0.1283 & 0.0728 & 0.0527 & 0.0674 & 0.0982 \\
\hline \multirow[t]{2}{*}{ BS, HV } & MAPE & 18.575 & 12.636 & 23.544 & 32.578 & 19.153 & 10.584 & 17.53 & 31.99 & 16.144 & 9.1181 & 16.355 & 28.024 \\
\hline & RMSE & 0.2509 & 0.154 & 0.2923 & 0.3972 & 0.2687 & 0.1335 & 0.2164 & 0.3949 & 0.2258 & 0.1141 & 0.2021 & 0.3409 \\
\hline \multirow[t]{2}{*}{ MBAW, HV } & MAPE & 14.2 & 7.7069 & 21.043 & 29.211 & 15.817 & 6.6303 & 15.217 & 29.409 & 12.509 & 4.8316 & 13.071 & 25.463 \\
\hline & RMSE & 0.2324 & 0.1248 & 0.2815 & 0.3823 & 0.2442 & 0.0939 & 0.1863 & 0.3715 & 0.1985 & 0.0713 & 0.1605 & 0.3142 \\
\hline \multirow[t]{2}{*}{ MQuad, HV } & MAPE & 14.392 & 7.6354 & 21.137 & 30.091 & 16.053 & 6.5843 & 15.282 & 30.084 & 12.707 & 4.7634 & 13.225 & 26.116 \\
\hline & RMSE & 0.2342 & 0.1236 & 0.2807 & 0.3875 & 0.2483 & 0.0934 & 0.1878 & 0.3787 & 0.2029 & 0.0709 & 0.163 & 0.322 \\
\hline
\end{tabular}




\section{ACKNOWLEDGEMENTS}

A part of this research is supported by 2006 Summer Research Fellowship of Pott College of Science and Engineering at the University of Southern Indiana.

\section{REFERENCES}

1. Barone-Adesi, Giovanni, and Robert E. Whaley, 1987, Efficient Analytic Approximation of American Option Values, Journal of Finance 42, 301-320.

2. Black, Fischer, and Myron S. Scholes, 1973, The Pricing of Options and Corporate Liabilities, Journal of Political Economy 81, 637-654.

3. Blomeyer, Edward C., 1986, An Analytical Approximation for the American Put Price for Options of Stocks with Dividends, Journal of Financial and Quantitative Analysis (June), 229-233.

4. Blomeyer, Edward C., and Herb Johnson, 1988, An Empirical Examination of the Pricing of American Put Options, Journal of Financial and Quantitative Analysis (March), 13-22.

5. Broadie, Mark, and Jerome B. Detemple, 2004, Option Pricing: Valuation Models and Applications, Management Science Vol. 50, No. 9, 1145-1177.

6. Cox, John C., Stephen A. Ross, and Mark Rubinstein, 1979, Option Pricing: A simplified Approach, Journal of Financial Economics 3, 229-263.

7. Ederington, L., and Guan, W., 2002, Measuring Implied Volatility: Is an Average Better? Which Average? Journal of Futures Markets 22, 811-837.

8. Geske, Robert, and H. E. Johnson, 1984, The American Put Valued Analytically, Journal of Finance 39, 1511-1524.

9. Geske, Robert, and Richard Roll, 1984, On Valuing American Call Options with the Black-Scholes European Formula, Journal of Finance 39, 443-455.

10. Ju, Nengjiu, and Rui Zhong, 1999, An Approximate Formula for Pricing American Options, Journal of Derivatives Vol. 7, No.2, 31-40.

11. Johnson, H., 1986, An Analytic Approximation for the American Put Price, Journal of Financial and Quantitative Analysis (June), 141-148.

12. Loudon, G. F., 1990, American Put Pricing: Australian Evidence, Journal of Business Finance \& Accounting 17, 297-321.

13. MacMillan, L. W., 1986, An Analytic Approximation for the American Put Option, Advances in Futures and Options Research 1, 119-139.

14. Merton, Robert C., 1973, The Relationship between Put and Call Option Prices: Comment, Journal of Finance 28, 183-184.

15. Merton, Robert C., 1973, The Theory of Rational Option Pricing, Bell Journal of Economics and Management Science, 4, 141-183.

16. Overdahl, James A., 1988, The Early Exercise of Options on Treasury Bond Futures, Journal of Financial and Quantitative Analysis 23, 437-449.

17. Shaw, William T., 1998, Modeling Financial Derivatives with Mathematica, Cambridge: United Kingdom, Cambridge University Press.

18. Stoll, Hans R., 1969, The Relationship between Put and Call Prices, Journal of Finance 24, 810-822.

19. Stampfli, Joseph G., and Victor Goodman, 2001, The Mathematics of Finance: Modeling and Hedging, The Brooks/Cole Series in Advanced Mathematics.

20. Whaley, Robert E., 1986, Valuation of American Futures Options: Theory and Empirical Tests, Journal of Finance 41, 127-150.

21. Zhu, Song-Pin, 2006, An exact and explicit solution for the valuation of American put options, Quantitative Finance, Vol. 6, Iss. 3, 229-242. 